University of Nebraska - Lincoln

DigitalCommons@University of Nebraska - Lincoln

Faculty Publications in Computer \& Electronics Electrical \& Computer Engineering, Department Engineering (to 2015)

2011

\title{
A Cross-Layer Parallel Handover Optimization Scheme for WiMAX Networks
}

Ting Zhou

University of Nebraska-Lincoln, tzhou@unInotes.unl.edu

Hamid Sharif

University of Nebraska-Lincoln, hsharif@unl.edu

Michael Hempel

University of Nebraska Lincoln, mhempel2@unl.edu

Puttipong Mahasukhon

University of Nebraska-Lincoln, pmahasukhon@huskers.unl.edu

Tao Ma

University of Nebraska Lincoln, tma@unlnotes.unl.edu

See next page for additional authors

Follow this and additional works at: https://digitalcommons.unl.edu/computerelectronicfacpub

Part of the Computer Engineering Commons

Zhou, Ting; Sharif, Hamid; Hempel, Michael; Mahasukhon, Puttipong; Ma, Tao; and Shrestha, Pradhumna, "A Cross-Layer Parallel Handover Optimization Scheme for WiMAX Networks" (2011). Faculty Publications in Computer \& Electronics Engineering (to 2015). 94.

https://digitalcommons.unl.edu/computerelectronicfacpub/94

This Article is brought to you for free and open access by the Electrical \& Computer Engineering, Department of at DigitalCommons@University of Nebraska - Lincoln. It has been accepted for inclusion in Faculty Publications in Computer \& Electronics Engineering (to 2015) by an authorized administrator of DigitalCommons@University of Nebraska - Lincoln. 


\section{Authors}

Ting Zhou, Hamid Sharif, Michael Hempel, Puttipong Mahasukhon, Tao Ma, and Pradhumna Shrestha 


\title{
A Cross-Layer Parallel Handover Optimization Scheme for WiMAX Networks
}

\author{
Ting Zhou, Hamid Sharif, Michael Hempel, Puttipong Mahasukhon, Tao Ma, Pradhumna Lal Shrestha \\ Computer and Electronics Engineering Department \\ University of Nebraska-Lincoln \\ Omaha, NE USA \\ \{tzhou, hsharif, mhempel,pmahasukhon, tma,plshrestha\}@ unlnotes.unl.edu
}

\begin{abstract}
The handover performance plays a crucial role in guaranteeing the quality of real-time applications in WiMAX networks. In general, a handover process can be divided into four stages: i) cell reselection, ii) handover preparation, iii) link layer handover, and iv) IP layer handover. A cross-layer parallel handover optimization (CPHO) scheme is proposed in this paper to reduce the handover signaling overhead and latency in each stage. The key idea of our proposed scheme is that uses the knowledge achieved from the backhaul inter-BS communications to reduce the HO control message load in wireless links and overlaps the executions of the link layer and the network layer handover process. Therefore the mean of the handover interruption time can be significantly reduced. The numerical analysis and simulation results show that the proposed approach significantly enhances the handover performance.
\end{abstract}

Keywords- Handover, WiMAX, Cross-layer Optimization

\section{INTRODUCTION}

A full end-to-end WiMAX network is defined by IEEE 802.16 standards [1] and WiMAX Forum Network Working Group (NWG) specifications [2]. The IEEE 802.16 standard defines the air interface specifications, including the physical (PHY) and medium access control (MAC) layers to support high speed data transmission and mobility service. The whole architecture reference model, reference points, protocols and procedures of WiMAX networks are described in WiMAX NWG specifications to ensure interoperability among different equipment vendors. WiMAX has been approved by ITU-T as a $3 \mathrm{G}$ standard since 2007, as well as UMTS and CDMA2000. Sprint, the third largest wireless carrier in North America, has launched WiMAX services in more than 30 major cities since 2009. Sprint's peak download speed is up to $12 \mathrm{Mbps}$ and the average downlink speed is about $2-4 \mathrm{Mbps}$.

Handover is the process of maintaining the service connection of a mobile station (MS) as it changes its point of attachment (PoA) to the access network. There are two categories of handover: the link layer (Layer-2) handover (L2HO) and the network layer (Layer-3) handover (L3HO). The L2HO only changes the physical access interface attachment point of an MS, while a L3HO involves changes in

Manuscript submitted at April 15th, 2011. This work was supported by a grant from the Federal Railroad Administration (FRA) under Grant 25-1105. Support was provided to us by BNSF and Union Pacific Railroad.

The authors are with the Telecommunications Engineering Laboratory (TEL), Department of Computer and Electronics Engineering, University of Nebraska-Lincoln, Omaha, NE 68182 USA (e-mail: hsharif@unl.edu).

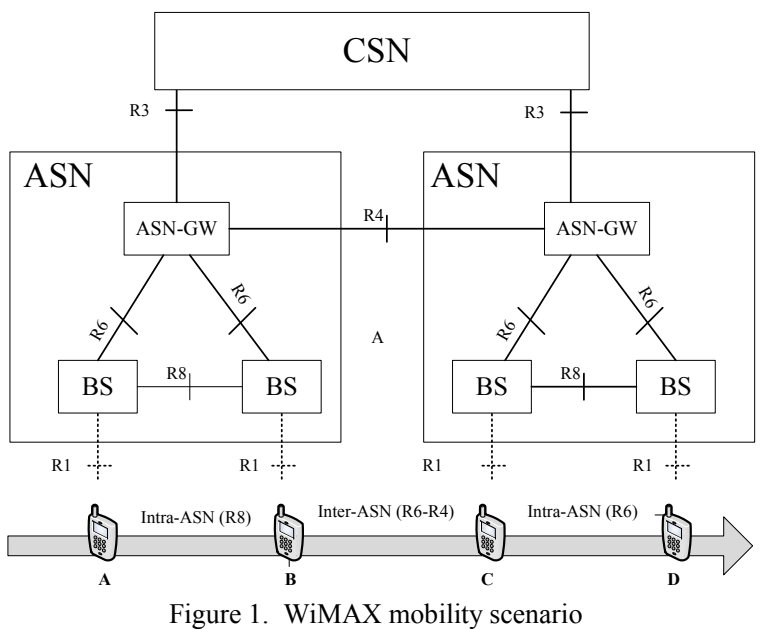

the IP configurations of the MS.

The architectural reference model of WiMAX networks, defined by the NWG, can be logically divided into two parts: Network Service Provider (NSP) and Network Access Provider (NAP). The core component of NSP is the Connectivity Service Network (CSN), which performs core network functions such as policy and admission control, IP address allocation, authentication and billing. It is also responsible for internetworking with non-WiMAX networks and for roaming through links to other NSPs. The NAP provides a complete set of link layer connectivity functions to WiMAX subscribers. It includes one or more Access Service Networks (ASNs). An ASN is composed of at least one BS and one gateway (ASN-GW). The WiMAX NWG Release 1 [6] defines three profiles that classify the distribution of functions among BS and ASN-GW. Profile C is supported by most device vendors, in which $\mathrm{HO}$ is controlled by the BS. Also, the Radio Resource Controller (RRC) is located at the $\mathrm{BS}$, while the ASN-GW is equipped with an RRC relay for delivering Radio Resource Management (RRM) messages among BSs.

The NWG has developed several open-interface reference points (RPs). R1 is the radio interface between BS and MS, which is defined in the IEEE 802.16 standard. Other RPs, from $\mathrm{R} 2$ to $\mathrm{R} 8$, are defined in NWG specifications. There are three kinds of mobility scenarios in WiMAX networks (Fig-1). $\mathrm{R} 6 / \mathrm{R} 4 \mathrm{HO}$ is referred to as inter-ASN HO, while R6 and R8 
HO belong to intra-ASN HO. Inter-ASN HO includes both $\mathrm{L} 2 \mathrm{HO}$ and $\mathrm{L} 3 \mathrm{HO}$, which involves the change of FAs.

In this paper, we focus on the optimization of the inter-ASN mobility scenario. First we analyze the inter-ASN handover process in WiMAX networks, and then propose our crosslayer parallel handover optimization (CPHO) scheme, which utilizes backhaul inter-BS communications and overlaps $\mathrm{L} 2 \mathrm{HO}$ and $\mathrm{L} 3 \mathrm{HO}$ executions to reduce cell reselection and handover execution latency. The key idea of CPHO is shown in Fig. 2. By reducing handover interruption, the QoS of realtime applications can be improved. Both theoretical analysis and NS-2 simulations show that our proposed approach can significantly enhance handover performance.

The remainder of this paper is structured as follows. In Section II, a detailed analysis of the handover process in WiMAX networks is provided and related research studies are reviewed. The architecture of our proposed cross-layer handover optimization approach is presented in Section III. Theoretical analysis and simulation validation are presented in Section IV and V respectively. Finally, Section VI concludes the paper.

\section{HANDOVER PROCESS ANALYSIS AND RELATED WORK}

To provide mobility services, the IEEE 802.16 standard specifies three different types of L2HO management techniques: hard handover (HHO), which is mandatory, macro-diversity handover (MDHO), and fast BS switching (FBSS). The implementation of HHO is simplest, however in which an MS suffers from service disruption until it completes the network re-entry process with the target BS. This results in usually a long interruption with negative impact on packet loss, and degrades the quality of the time sensitive real-time applications. In MDHO and FBSS, an MS can register with several base stations (BS) simultaneously, as long as they share the same carrier frequency and a common timing source. They can achieve less handover latency at the expense of network overhead, implementation complexity and limited frequency reuse.

Two L3HO management types, Client Mobile IP (CMIP) [3] and Proxy Mobile IP (PMIP) [4], are optionally supported in WiMAX NWG. In the CMIP mechanism, an MS registers on foreign networks and connects back to its home network via a combination of Foreign Agents (FAs) and Home Agents (HAs). When an MS moves to another network, it will detect a new FA, obtain a Care-of-Address (CoA), and then register this CoA with the HA. The CoA is used by the HA to tunnel and forward packets to the MS directly. With PMIP, an MS gets a new IP address by DHCP [5] when it moves to another network. The HA does not forward packets directly to the MS, but sends them to an FA in the visited network. The FA is responsible for delivering packets to the MS. In CMIP, the MIP stack exists in an MS, increasing complexity. Also, CMIP is incompatible with IP-sec based virtual private networks (VPNs) and network address translator (NAT) gateways. In PMIP, it is not necessary to implement a client MIP stack in MS, simplifying the MS implementation. However, because PMIP is dependent on FAs to relay packets, it requires more

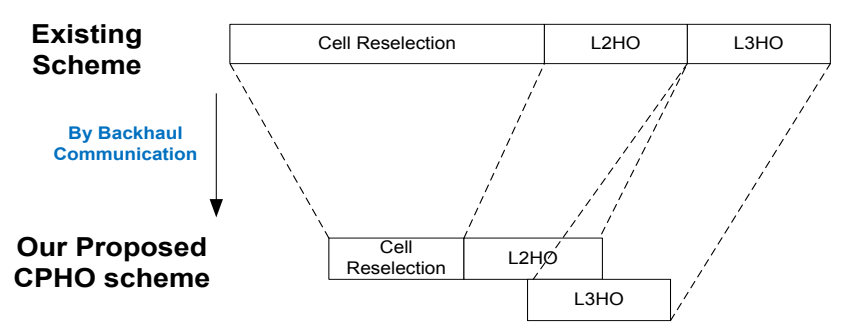

Figure 2. Key idea of our proposed CPHO scheme

network overhead than CMIP. During L3HO, the MS must reregister with $\mathrm{HA}$ and bind with FA, so it causes longer handoff latency and higher packet loss than with L2HO. Fast Mobile IPv6 (FMIPv6) [6] is designed by Internet Engineering Task Force (IETF) to improve handover performance over Mobile IPv6 in assistance with Layer-2 event triggers.

A handover may be initiated by either BSs or MSs. An MS may initiate a handover when it detects that the received radio signal from its serving BS deteriorates or experiences interference in the current frequency band. A BS may initiate a handover to balance an uneven distribution of MS traffic.

A HHO process in WiMAX networks can generally be divided into four stages: i) cell reselection; ii) $\mathrm{HO}$ initiation and preparation; iii) L2HO execution; and iv) L3HO execution.

Cell reselection refers to the process of collecting the information of candidate target BSs by scanning and/or associating with one or more BSs to determine their suitability. At the $\mathrm{HO}$ initiation and preparation stage, the MS decides on triggering $\mathrm{HO}$ from the serving $\mathrm{BS}$ to the target $\mathrm{BS}$ while the serving BS is responsible for releasing current connections and resources.

A few existing 802.16 HO-enhanced schemes focus on the optimization of the cell reselection stage to accurately predict the HO target BS and reduce the number of scanned channels. By measuring the human mobility patterns for two months, the author in [7] found that the most popular destination pair is home to work (office or school) and vice versa followed by home to mall and work to food or pubs. Also, about $90 \%$ of the time people choose the same route. The authors therefore assumed that with previously acquired knowledge of human mobility behavior, an MS can maintain a sequence of BS IDs to which it connects while on a trip. This way, the target BSs can be chosen before $\mathrm{HO}$ is sought.

Other existing HO-optimized schemes focus on either the L2HO or L3HO process. A passport handover scheme was proposed in [8] to accelerate the Layer-2 handover process. In this scheme, the connection CIDs assigned by the serving BS are accepted by the handover target $\mathrm{BS}$ during the handover process until new CIDs are assigned by an REG-RSP message. However, without inter-BS communications, to avoid old CIDs, which are carried by the handover MS to collide with existing CIDs in the target cell, the passport handover scheme marks a certain reserved 3-bit-length in a 16-bit CID to satisfy the traditional $1 / 7$ frequency reuse strategy. [9] proposed a mechanism that optimizes the FMIPv6 handover procedure with the assistance of IEEE 802.21 [10] MIH services for vehicular networking. The main improvements include 


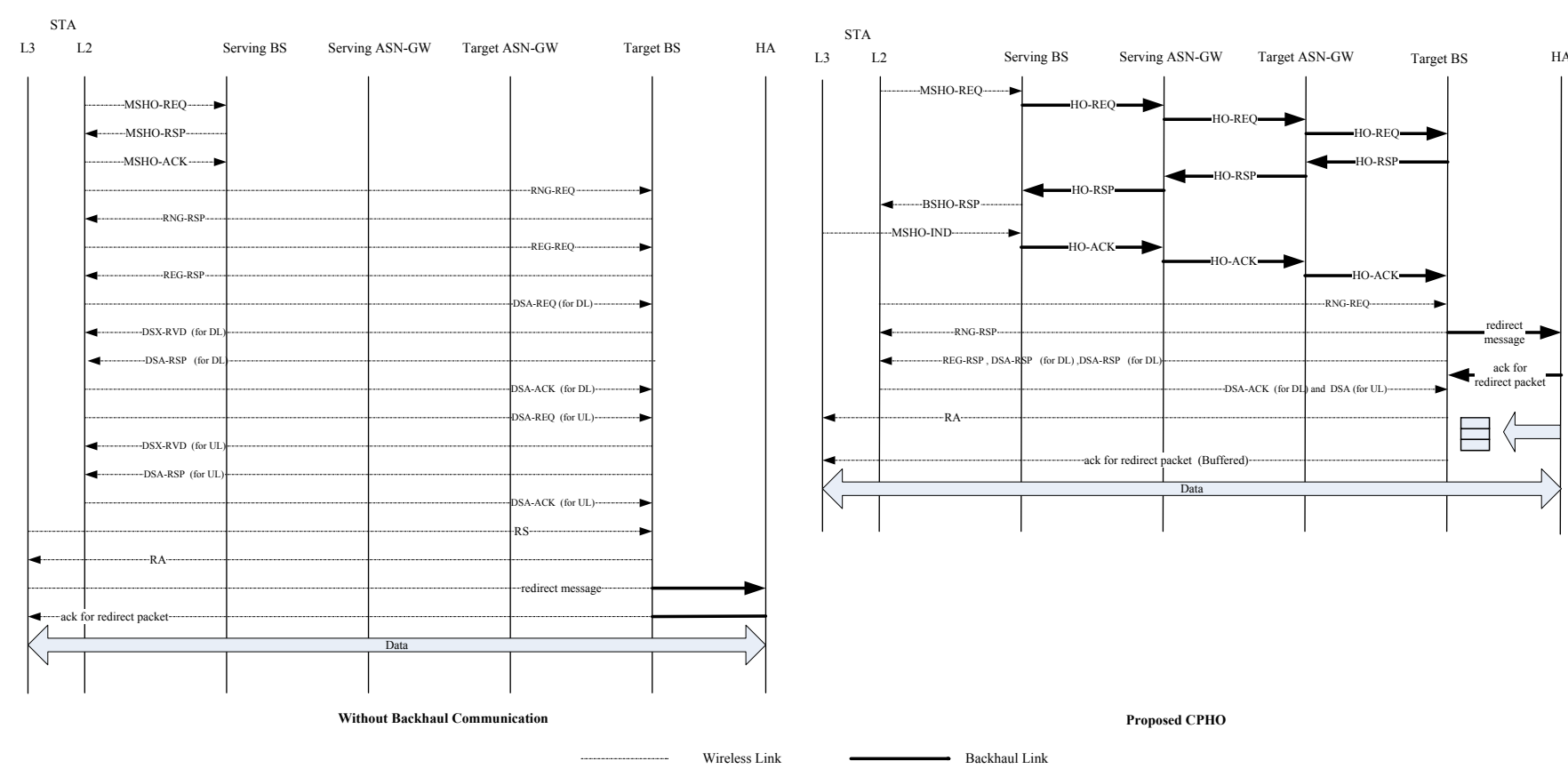

Figure 3. Message flow comparison between the existing scheme and our proposed CPHO scheme

broadcasting Layer-2 and Layer-3 neighboring access network information together, and storing the latest received routing messages in the cache of the MS to reduce signaling overheads and the long anticipation time imposed by MIPv6.

To the best of our knowledge, our proposed CPHO scheme is the first one that optimizes each stage's performance in the handover process by redistributing handover message load from the wireless links to the wired backhaul links, as well as using the backhaul inter-BS communications information to overlap the $\mathrm{L} 2 \mathrm{HO}$ and $\mathrm{L} 3 \mathrm{HO}$ executions.

\section{PROPOSED CROSS-LAYER PARALLEL HANDOVER OPTIMIZATION SCHEME}

The framework of the proposed CPHO scheme is described in detail as follows:

1) The BSs periodically broadcast an MOB_NBR-ADV message. According to this message, an MS can get the neighbor BS list of its serving BS.

2) An MS will come into the cell selection stage when the signal strength of its serving $\mathrm{BS}$ is reduced and is less than the given threshold.

3) At the cell selection stage, Association Level 2 (Network Assisted Association Reporting) is used to collect the information of candidate target BSs. Before channel scanning, the SS sends an MOB_SCN-REQ message to the serving BS, which includes a list of the candidate target BSs. Then the serving BS negotiates with each target BS via backhaul networks to assign the MS a dedicated ranging region, and transmits the result to the MS by an MOB_SCN-RSP message. Because the ranging response is sent via the backhaul network, the MS does not have to wait for the response from the target BS after sending the RNG-REQ message and can switch to the next channel immediately. The serving BS aggregates all ranging-related information into a single MOB_ASCREPORT message, and then forwards it to the MS.

4) In accordance with the information collected from the cell selection stage, an $\mathrm{HO}$ decision is made for the MS to switch from the serving BS to the $\mathrm{HO}$ target BS.

5) The MS notifies the serving BS about its decision to perform an $\mathrm{HO}$ by issuing an MOB_MSHO-REQ message, which includes the ID of the $\mathrm{HO}$ target BS and the QoS requirement to the serving BS. The serving BS sends an $\mathrm{HO}-$ REQ through the backhaul network via R4 and R6 paths to the target BS. The target BS responds by sending an HO-RSP message to the serving BS, which assigns the MS a dedicated ranging region, including the downlink (DL) and the uplink (UL) synchronization parameters such as ranging status, timing offset adjustment, power level adjustment and frequency offset adjustment. The serving BS encapsulates the above information into an MOB_BSHO-RSP message, and then forwards it to the MS. The MOB_HO-IND message, sent by the MS, integrates link layer requests, including REG-REQ and DSA-REQs for DL/UL in both directions and IP layer requests which include the Router Solicitation (RS) and the redirect message together. When receiving this MOB_HOIND message, the serving BS encapsulates its payload into a HO-ACK message and sends it to the HO target BS. Through the above inter-BS communications through the backhaul network, the MS achieves the physical layer and MAC layer synchronization parameters of the target BS before the L2HO execution.

6) The HO target BS relays the redirect message to the HA, and then stores the redirect acknowledgement, which is sent by the HA into its cache.

7) The MS switches to the frequency of the HO target BS, and then uses the dedicated UL time slot to send the RNG-REQ message and wait for the RNG-RSP. The HO target BS sends 

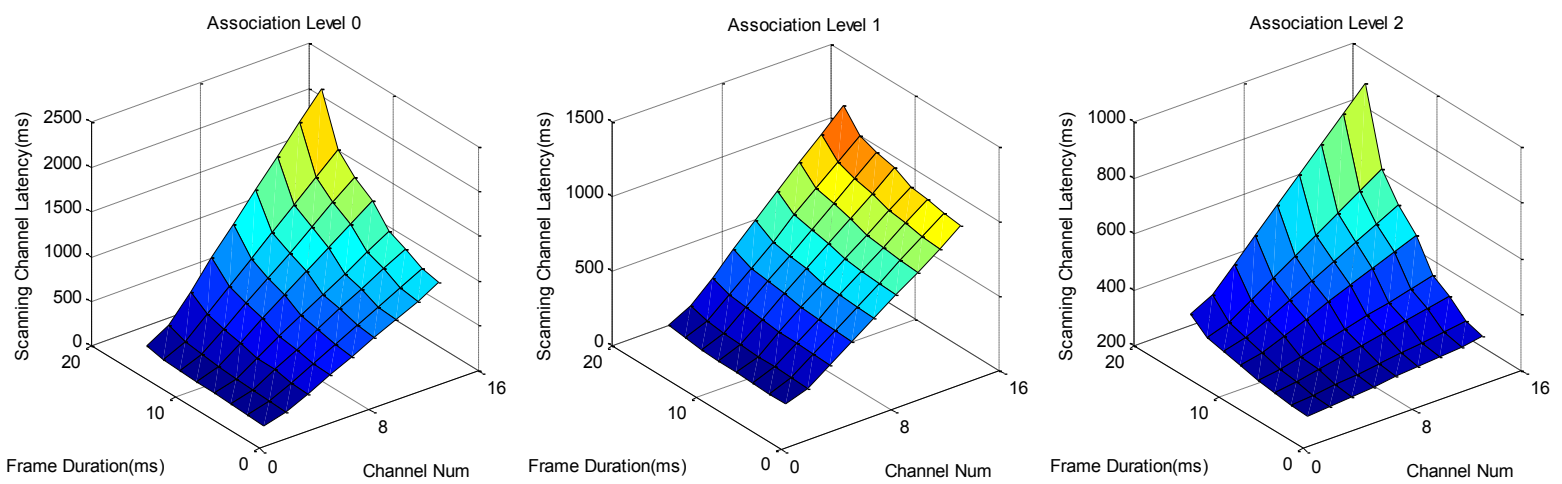

Figure 4. The relationship between scanning channel latency and association levels

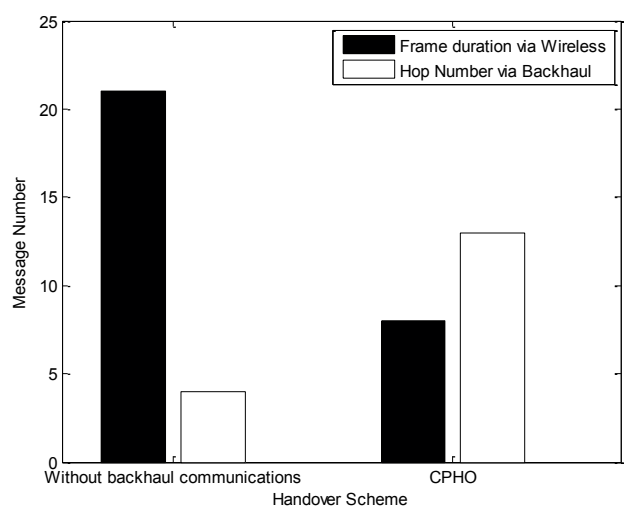

Figure 5. Message distributions for $\mathrm{CPHO}$ and the existing scheme

RNG-RSP, REG-RSP and bidirectional DSA-RSPs in one DL burst to the MS. DSA-ACKs for both DL/UL directions are sent by the MS in response to the received DSA-RSPs.

8) The target BS sends both the Router Advertisement (RA) and the buffered redirect acknowledgement to the MS to complete the entire $\mathrm{HO}$ process. The MS set this target BS as its current serving BS.

Fig. 3 shows the message exchange flow comparison between our proposed $\mathrm{CPHO}$ and the existing $\mathrm{HO}$ scheme without backhaul inter-BS communications.

\section{THEORETICAL ANALYSIS}

A HHO process in WiMAX networks can generally be divided into four stages: 1) cell reselection; 2) HO initiation and preparation; 3) L2HO execution; and 4) L3HO execution. In the rest of this section, we analyze the latency in each stage latency of the existing $\mathrm{HO}$ scheme without backhaul inter-BS communications, and compare it with that of our proposed $\mathrm{CPHO}$ scheme.

\section{1) Cell reselection}

The MS may get the information of candidate target BSs by incorporating MOB_NBR-ADV messages that are periodically broadcasted by its serving BS. There are three scan/ associating modes defined in the IEEE 802.16 standard: Association Level 0,1 and 2. Without inter-BS backhaul communication, only Association Level 0 can be used for channel scanning.

1) Association Level 0 (Association without coordination): The serving BS only allocates periodic intervals to the MS to scan channels. The target BS has no knowledge of the MS and provides only contention-based ranging allocations. If $\mathrm{M}$ is the maximum number of contention slots that an MS needs to wait before sending the ranging request, $\mathrm{n}_{c s}$ is the number of contention slots per frame. If an MS switches to channel $i$, it needs to detect the preamble of the DL frame of the target BS $B S_{i}$ in that channel, and use it to synchronize with $B S_{i}$. Let $D_{i}$ be the frame duration of $B S_{i}$, and then the channel scanning time in channel $i$ is

$$
T s_{l 0_{-} i}=\frac{k}{n_{c s}} D_{i}+t_{\text {syn }}+t_{\text {timeout }}
$$

where $\mathrm{k}$ is the random value between $[0, \mathrm{M}], t_{s y n}$ is synchronization time, and $t_{\text {timeout }}$ is the maximum wait time for the ranging response frame from $B S_{i}$. In [11], $t_{\text {timeout }}$ is set to $50 \mathrm{~ms}$.

If all BSs have the same frame duration $D_{f}$, there are a total $\mathrm{N}$ channels that need to be scanned, and the synchronization time is also the same, then the average total channel scanning time is

$$
E\left[T_{s_{-} l 0}\right]=N\left(\frac{M}{2 n_{c s}} D_{f}+t_{\text {syn }}+t_{\text {timeout }}\right)
$$

where E[.] is the expectation operator. It is clear that $\mathrm{E}\left[\mathrm{T}_{\mathrm{S}_{1} 10}\right]$ shows a linear growth with $\mathrm{N}$.

2) Association Level 1 (Association with coordination): Before scanning the channel, the BS sends an MOB_SCNREQ message to the serving BS, which includes the waiting list of the target BSs. Then, the serving BS negotiates with each target BSs via backhaul networks to assign the MS a dedicated ranging region, and transmits the result to the MS by an MOB_SCN-RSP message.

Therefore, the total channel scanning time in this mode is

$$
T_{s_{-} l 1}=t_{m s \leftrightarrow s b s}+\operatorname{MAX}_{i \in N}\left(t_{s b s \leftrightarrow t b s_{i}}\right)+\sum_{i}^{N}\left(t_{s y n}+t_{\text {timeout }}\right)
$$

where $t_{m s \leftrightarrow s b s}$ is the communication delay between the MS and the serving BS, and $\mathrm{t}_{s b s \leftrightarrow t b s_{i}}$ is the communication delay 
TABLE-1. SIMULATION PARAMETERS

\begin{tabular}{|c|l|}
\hline Cell Layout (hexagonal cell) & 7 \\
\hline Number of BS & 10 \\
\hline Number of ASN-GW & 2 \\
\hline Number of MS & 1 \\
\hline 802.16 Bandwidth (MHz) & 10 \\
\hline 802.16 FFT Size & 1024 \\
\hline 802.16 Frame Duration (msec) & 10 \\
\hline 802.16 Synchronization Delay (msec) & 10 \\
\hline 802.16 Ranging Timeout (msec) & 50 \\
\hline 802.16 Maximum Contention Window & 5 \\
\hline MS Speed (m/s) & 5 \\
\hline Wired Per-hop Delay (msec) & 5 \\
\hline Hop Number between BS and HA & 2 \\
\hline VolP Packet Size (msec) & 160 \\
\hline VolP Traffic Rate (kbps) & 400 \\
\hline
\end{tabular}

between the MS and the serving BS between the MS and the target $B S_{i}$.

3) Association Level 2 (Network Assisted Association Reporting): the procedure is similar to Level 1 except that the MS does not wait for a response from the target BS. The ranging response is sent by the target $\mathrm{BS}$ to the serving $\mathrm{BS}$ via the backhaul network. The serving BS aggregates all rangingrelated information into a single MOB_ASC-REPORT message, and then forwards it to the MS.

The total channel scanning time in this mode is

$$
\begin{aligned}
& T_{s_{-} l 2}=t_{s y n}+t_{m s \leftrightarrow s b s}+\underset{i \in N}{M A X}\left(t_{s b s \leftrightarrow t b s_{i}}\right)+ \\
& \sum_{i}^{N}\left(t_{s y n}+D_{i}\right)+M_{i \in N} A X\left(t_{t b s_{i} \leftrightarrow s b s}\right)+t_{s b s \leftrightarrow m s}
\end{aligned}
$$

where $t_{s b s \leftrightarrow m s}$ is the MOB_ASC-REPORT message transmit time, and $\mathrm{t}_{t b s_{i} \leftrightarrow s b s}$ is the time that each target $B S_{i}$ sends the ranging response to the serving BS.

Assuming that all BSs have the same frame duration $\mathrm{D}_{\mathrm{f}}$, $\mathrm{t}_{m s \leftrightarrow s b s} \approx \mathrm{t}_{s b s \leftrightarrow m s}=\mathrm{D}_{\mathrm{f}}, \mathrm{t}_{t b s_{i} \leftrightarrow s b s} \approx \mathrm{t}_{s b s \leftrightarrow t b s_{i}}$, and the maximum backhaul communication delay is $100 \mathrm{~ms}$, the scanning channel latency comparison among three association levels is shown in Fig. 4. It is clear that using backhaul communications, Association Level 2 has the lowest scanning channel latency. If we cannot change the frame duration, a reduction in the total number of scanning channels will efficiently reduce the scanning channel latency. In the proposed CPHO scheme, Association Level 2 is chosen for channel scanning.

\section{2) HO initiation and preparation}

Based on the information collected from the cell selection stage, the MS chooses the most suitable candidate BS as its $\mathrm{HO}$ target BS.

In the existing scheme without integrating with the backhaul inter-BS communications, the MS only notifies the serving BS for releasing current connections and resources by exchanging Mobile Scan Request (MOB_MSHO-REQ) and Mobile Scan Response (MOB_SCN-RSP). The MS then transmits MOB $\mathrm{HO}-\mathrm{IND}$ to notify the serving $\mathrm{BS}$ to commence the $\mathrm{HO}$ execution. Therefore, for the existing scheme, $\mathrm{t}_{\text {p,existing }}$ the latency in this stage is

$$
T_{p \text {,existing }}=2 t_{m s \leftrightarrow s b s}+t_{s b s \leftrightarrow m s}
$$

In the proposed $\mathrm{CPHO}$ scheme, the MS use the three-way handshake to communicate with the target $\mathrm{BS}$ via the backhaul links, and thus the latency is

$$
T_{p, C H P O}=2 t_{m s \leftrightarrow s b s}+t_{s b s \leftrightarrow m s}+2 t_{s b s \leftrightarrow t b s}+t_{t b s \leftrightarrow s b s}
$$

\section{3) $\mathrm{L} 2 \mathrm{HO}$ execution}

In the existing scheme without backhaul communications, after the MS switches to the frequency of the target BS and discovers the initial ranging slot by the receiving ULMAP to initiate the process of ranging, the MS has to pipeline execute an RNG-REQ/RSP exchange, an REG-REQ/RSP exchange, and two 4-way DSA handshakes. Given $\mathrm{t}_{\text {L2, existing, the }}$ latency in L2HO stage is

$$
T_{L 2, \text { existing }}=\frac{k}{n_{c s}} D_{f}+t_{s y n}+6 t_{m s \leftrightarrow t b s}+6 t_{t b s \leftrightarrow m s}
$$

where $\mathrm{k}$ is the random value between $[0, \mathrm{M}]$.

However in the proposed CPHO scheme, the MS has required the dedicated ranging slot and has sent REG-REQ and DSA-REQs to the target BS via the backhaul links in the previous stage, and so the REG-RSP and DSA-RSPs can be transmitted together in one DL burst. Therefore the latency can be reduced to

$$
T_{L 2-A, C P H O}=2 t_{m s \leftrightarrow t b s}+2 t_{t b s \leftrightarrow m s}
$$

Meanwhile the target BS relays the redirect message to the MS's HA and waits for the redirect acknowledgement from the HA. The latency is

$$
T_{L 2-B, C P H O}=t_{t b s \leftrightarrow H A}+t_{H A \leftrightarrow t b s}
$$

The total CPHO latency in this stage is

$$
T_{L 2, \text { CPHO }}=\operatorname{MAX}\left(T_{L 2-A, C P H O}, T_{L 2-B, C P H O}\right)
$$

4) $\mathrm{L} 3 \mathrm{HO}$ execution

In this stage, the existing scheme has to pipeline execute an $\mathrm{RS} / \mathrm{RA}$ handshake between the MS and the target BS, and a redirect message handshake between the MS and the HA. Thus the latency is

$$
T_{L 3, \text { existing }}=2 t_{m s \leftrightarrow t b s}+2 t_{t b s \leftrightarrow m s}+t_{t b s \leftrightarrow H A}+t_{H A \leftrightarrow t b s}
$$

where $\mathrm{t}_{t b s \leftrightarrow H A}$ and $\mathrm{t}_{H A_{i} \leftrightarrow t b s} \mathrm{a}$ are the unidirectional delay from the target $\mathrm{BS}$ to the HA or vice versa.

In this stage, the proposed CPHO scheme only needs to transmit two downlink direction packets, which can be transmitted together, and so the latency in this stage is

$$
T_{L 3, \text { CPHO }}=t_{t b s \leftrightarrow m s}
$$

Assuming $\mathrm{t}_{t b s \leftrightarrow \mathrm{HA}} \approx \mathrm{t}_{H A \leftrightarrow \mathrm{tbs}}, \mathrm{t}_{m s \leftrightarrow t b s} \approx \mathrm{t}_{t b s \leftrightarrow \mathrm{ms}}$, and the mobility scenario is the same as with Fig. 1. The transmit frame duration number via the wireless links and the transmit hop number via the wired links comparison between the existing scheme and the proposed CPHO are shown in Fig. 5.

\section{SimUlation VALIDATION}

The proposed CPHO scheme has been implemented in the NS-2 network simulator [12] based on the National Institute of Standards and Technology (NIST) WiMAX module [13]. To simplify the scenario, every BS supports FA and access router functions, in which one BS presents a different subnet. The bidirectional VoIP traffic is established between HA and MS. 


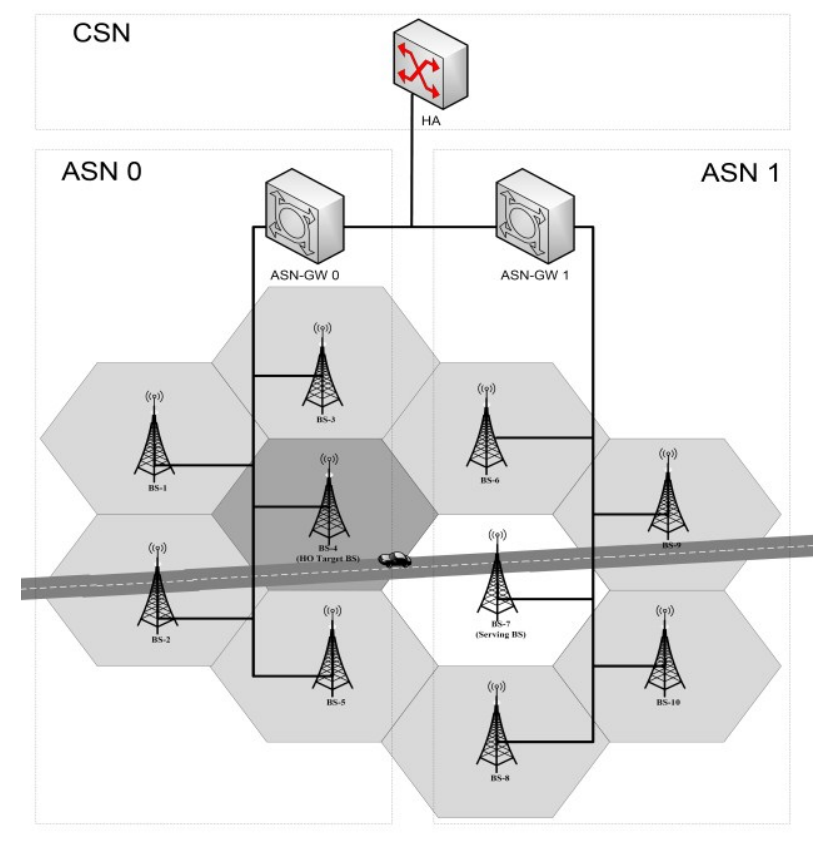

Figure 6. Inter-ASN simulation scenario

The simulation scenario is shown in Fig. 6. The Inter-ASN handover performances were measured when the MS moved between BS-7 and BS-4 along the road. The frequency reuse factor is 1/7. Table-1 lists the main parameters of our simulations.

Fig. 7 shows the average latency comparison between the proposed $\mathrm{CPHO}$ and the existing $\mathrm{HO}$ scheme without backhaul inter-BS communications. It is clear that in the HO initiation stage, CPHO brings a little longer latency than the existing scheme because of an additional inter-BS backhaul communication process. However, it can greatly reduce the latencies in other stages. These results validate that the proposed $\mathrm{CPHO}$ scheme can efficiently reduce the handover interruption by reducing the number of messages in wireless links and overlap the $\mathrm{L} 2 \mathrm{HO}$ and $\mathrm{L} 3 \mathrm{HO}$ executions.

\section{CONCLUSION}

Hard handover is a mandatory feature and must be supported in WiMAX networks. However, the long interruption of hard handover degrades the service quality of delay-sensitive applications. In this paper, a cross-layer parallel handover optimization scheme is proposed to reduce the handover latency by redistributing the handover message load from the wireless links to the wired backhaul links and executing the linker layer handover and network layer handover processes simultaneously. Through backhaul interBS communications, the MS can achieve the physical layer and the linker layer synchronization parameters of the target BS before the handover execution; therefore the L2HO latency can be reduced. Meanwhile, because the target BS has the linker layer and IP layer information of MSs before the handover execution, it can overlap the $\mathrm{L} 2 \mathrm{HO}$ and $\mathrm{L} 3 \mathrm{HO}$ processes. The numerical analysis and simulation results show that the proposed CPHO scheme significantly reduces the handover latency.

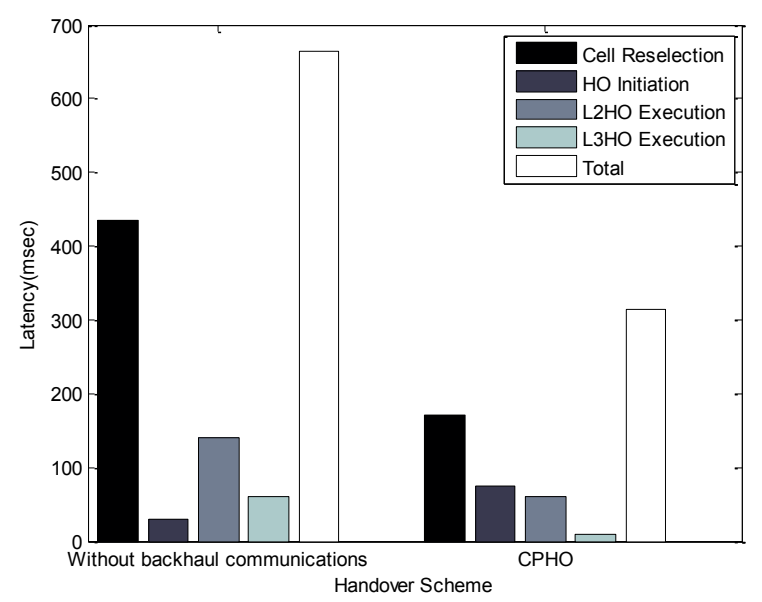

Figure 7. Handover latency simulation result

\section{ACKNOWLEDGMENTS}

This study was conducted by the Advanced Telecommunications Engineering Laboratory (TEL) (www.TEL.unl.edu ) researchers at the University of NebraskaLincoln and funded by the Federal Railroad Administration (FRA) under the direction of Terry Tse. This study would not have been possible without the excellent support from Union Pacific Railroad, BNSF Railway, and the Association of American Railroads (AAR).

\section{REFERENCES}

[1] IEEE Std 802.16-2009, "IEEE Standard for Local and metropolitan area networks Part 16: Air Interface for Broadband Wireless Access Systems," (Revision of IEEE Std 802.16-2004), May 292009.

[2] WiMAX Forum, www.wimaxforum.org

[3] Gundavelli, S., Leung, K., Devarapalli, V., Chowdhury, K., and B. Patil,, "Mobility Support in IPv6," IETF RFC 3775, June 2004.

[4] Johnson, D., Perkins, C., and J. Arkko, "Proxy Mobile IPv6," IETF RFC 5213, August 2008.

[5] R. Droms, "Dynamic Host Configuration Protocol," IETF RFC 2131, March 1997.

[6] R. Koodli et al., "Fast Handovers for Mobile IPv6," IETF RFC 4608, April 2006.

[7] Sachin Lal Shrestha, Nah-Oak Songt, and Song Chong, "Seamless Realtime Traffic Handover Policy for IEEE 802.16m Mobile WiMAX," 43rd Annual Conference on Information Sciences and Systems(CISS), pp. 252-257, March 2009.

[8] Wenhua Jiao , Pin Jiang, Yuanyuan Ma, "Fast Handover Scheme for Real-Time Applications in Mobile WiMAX," IEEE International Conference on Communications(ICC'07), pp. 6038-6042, June 2007.

[9] Qazi Bouland Mussabbir, Wenbing Yao, Zeyun Niu, and Xiaoming Fu, "Optimized FMIPv6 Using IEEE 802.21 MIH Services in Vehicular Networks," IEEE Transactions on Vehicular Technology, vol.56, no.6, pp. 3397-3407, November 2007.

[10] IEEE P802.21/D14.0, "Media Independent Handover Services," September 2008.

[11] Richard Rouil and Nada Golmie, "Adaptive Channel Scanning for IEEE 802.16e," IEEE MILCOM 2006, pp.1-6, October 2006.

[12] NS-2, http://isi.edu/nsnam/ns/.

[13] NIST Seamless and Secure Mobility tool suite, http://www.nist.gov/itl/antd/emntg/ssm_tools.cfm 